\title{
Novel insights into the rehabilitation of memory post acquired brain injury: a systematic review
}

\author{
Lauriane A. Spreij ${ }^{1}$, Johanna M. A. Visser-Meily ${ }^{2}$, Caroline M. van Heugten ${ }^{3}$ and \\ Tanja C. W. Nijboer ${ }^{1,2 *}$ \\ ${ }^{1}$ Department of Experimental Psychology, Helmholtz Institute, Utrecht University, Utrecht, Netherlands \\ ${ }^{2}$ Brain Center Rudolf Magnus, Center of Excellence for Rehabilitation Medicine, University Medical Center Utrecht, and De Hoogstraat Rehabilitation, Utrecht, \\ Netherlands \\ ${ }^{3}$ Department of Psychiatry and Neuropsychology, Faculty of Health, Medicine and Life Sciences, Maastricht University, Maastricht, Netherlands
}

\section{Edited by:}

Francesco Di Russo, University of Rome Foro Italico, Italy

Reviewed by:

Arun Bokde, Trinity College Dublin, Ireland

Rocco Salvatore Calabrò, IRCCS

Centro Neurolesi Bobibo-Pulejo, Italy

*Correspondence:

Tanja C. W. Nijboer, Department Experimental Psychology, Hemholtz Institue, Utrecht University,

Heidelberglaan 2, 3584 CS Utrecht,

Netherlands

e-mail: t.c.w.nijboer@uu.nl
Objective: Acquired Brain Injury ( $\mathrm{ABI}$ ) frequently results in memory impairment causing significant disabilities in daily life and is therefore a critical target for cognitive rehabilitation. Current understanding of brain plasticity has led to novel insights in remediation-oriented approaches for the rehabilitation of memory deficits. We will describe 3 of these approaches that have emerged in the last decade: Virtual Reality (VR) training, Computer-Based Cognitive Retraining (CBCR) and Non-Invasive Brain Stimulation (NBS) and evaluate its effectiveness.

Methods: A systematic literature search was completed in regard to studies evaluating interventions aiming to improve the memory function after $A B I$. Information concerning study content and reported effectiveness were extracted. Quality of the studies and methods were evaluated.

Results: A total of 786 studies were identified, 15 studies met the inclusion criteria. Three of those studies represent the VR technique, 7 studies represent CBCR and 5 studies NBS. All 3 studies found a significant improvement of the memory function after VR-based training, however these studies are considered preliminary. All 7 studies have shown that $\mathrm{CBCR}$ can be effective in improving memory function in patients suffering from $\mathrm{ABI}$. Four studies of the 5 did not find significant improvement of the memory function after the use of NBS in ABI patients.

Conclusion: On the basis of this review, CBCR is considered the most promising novel approach of the last decade because of the positive results in improving memory function post $A B I$. The number of studies representing VR were limited and the methodological quality low, therefore the results should be considered preliminary. The studies representing NBS did not detect evidence for the use of NBS in improving memory function.

Keywords: acquired brain injury, memory, remediation-oriented technique, virtual reality, computer-based cognitive retraining, non-invasive brain stimulation

\section{INTRODUCTION}

Memory impairment is a common consequence of Acquired Brain Injury (ABI) which causes significant disabilities and is therefore a critical target for cognitive rehabilitation (Hall et al., 2005; Yip and Man, 2013). ABI is defined as damage to the brain that occurs after birth and is not related to congenital disorders, developmental disabilities, or processes that progressively injure the brain. The majority of $\mathrm{ABI}$ is caused by Traumatic Brain Injury (TBI) and hemorrhagic or ischemic stroke (Holmqvist

Abbreviations: ABI, Acquired Brain Injury; TBI, Traumatic Brain Injury; WM, Working Memory; VR, Virtual Reality; CBCR, Computer-Based Cognitive Retraining; NBS, Non-Invasive Brain Stimulation; TMS, Transcranial Magnetic Stimulation; rTMS, repetitive Transcranial Magnetic Stimulation; tDCS, transcranial Direct Current Stimulation. et al., 2009). Within the TBI population, the percentage of people suffering from some form of memory impairment ranges from 20 to $79 \%$, depending on the severity of the (closed) head trauma, the time of measurement, and the instruments used. Even after 1 year, 4 to $25 \%$ of TBI patients show some form of memory impairment (Cappa et al., 2011). The prevalence of memory dysfunction post-stroke varies from 23 to $55 \%$ in the first 3 months, which declines 1 year post-stroke to a percentage between 11 and 31\% (Das Nair and Lincoln, 2007; Snaphaan and de Leeuw, 2007; Aben et al., 2013). Memory impairments can hamper independence in activities of daily living, as well as return to work, social participation and the overall quality of life (Fish et al., 2008). For this reason and the high prevalence of memory impairment after ABI, there is a urgent need for effective cognitive rehabilitation. 
There are 2 main approaches within memory rehabilitation. First, remediation by restoration or retraining of the function; and second, compensation referring to any compensatory strategies, environmental modifications, and intact cognitive functions to overcome limitations in daily life. Remediation of the function is primarily due to some degree of spontaneous recovery (Cramer, 2008). The understanding of spontaneous recovery has been accompanied by the development of a wide range of therapeutic approaches that target brain repair by restoration. These can be referred to as remediation-oriented therapies, the aim of which is not to salvage threatened tissue but to promote restoration of function. Retraining of the function is based on the assumption that impaired memory will respond to mental exercise in the same manner as muscles respond to physical exercise and that repetitive training in 1 memory task may generalize to improved performance on other tasks within the same memory system (Brooks and Rose, 2003). The hypothesis is that the capacity of the function improves if the training is successful and does not depend on context or learning abilities (Björkdahl et al., 2013). Unfortunately, until recently there was little empirical evidence to indicate that these techniques are of much benefit to patients as any improvement on specific tasks practiced have not been found to generalize to other similar tasks (Brooks and Rose, 2003; Rees et al., 2007).

On the contrary, Rees et al. (2007) found strong evidence for the use of compensation for lost or deficient memory function. Therefore, most memory rehabilitation interventions focus on alleviating memory problems on functional level (i.e., level of activity in daily life), without necessarily improving the underlying memory function (Rees et al., 2007). Current memory treatment programs have focused on teaching patients the use of internal strategies (e.g., repeating, counting, face-name associations, categorizing, mental visualization or rhyming mnemonics) and/or external strategies (e.g., diaries, notebooks, to-do lists, electronic organizers, pagers) to help remembering and recalling information (Fish et al., 2008). In an updated review of evidencebased rehabilitation, Cicerone et al. (2011) recommended training in the use of external compensation strategies (including assistive technology) with direct application to daily activities as a practice guideline for individuals with moderate to severe memory impairment after TBI or stroke.

In brief, there has been little research showing that memory can be improved through remediation-oriented therapies and hence compensation approaches are the treatment choice. However, with the recently maturing fields in cognitive neurosciences, neuroplasticity shows greater promises then previously assumed and has yielded new interdisciplinary approaches (Miniussi et al., 2008). Neuroplasticity is the ability of the brain to create, strengthen, and modify neurological connections. It occurs at many levels from molecules to cortical reorganization. Remediation-oriented rehabilitation, based on neuroplasticity, can not only modify neural connections, but can also lead to functional relearning (Kimberley et al., 2010). This allows brain injured patients to relearn new knowledge and establish new skills (Li et al., 2013).

The current understanding of brain plasticity has led to novel insights in the rehabilitation of memory deficits. However, an overview of these insights is missing. The aim of this systematic review is to describe novel memory rehabilitation interventions based on remediation-oriented techniques post $\mathrm{ABI}$ and evaluate its effectiveness. This review will not include studies evaluating pharmacological intervention as pharmacological therapies were considered not suitable for targeting only the memory function without affecting other cognitive functions. We will describe 3 non-pharmacological approaches aiming at restoring the memory function that have emerged in the last decade: Virtual Reality (VR) training, Computer-Based Cognitive Retraining (CBCR) and Non-Invasive Brain Stimulation (NBS).

\section{VIRTUAL REALITY}

Virtual environments represent many real-life situations and are programmed to record accurate measurements of the individual's performance assessing the underlying function (Brooks and Rose, 2003). VR is an interactive computer technology which creates the illusion of being in an artificial world. An fMRI study indicated that virtual-based environments are able to activate the related brain parts as in the real environment (You et al., 2005). The transfer of learned skills from VR training to real-life situations has been reported, which shows a high ecological validity (Brooks and Rose, 2003). VR is often used to obtain a realistic and controlled assessment of memory impairment in a rehabilitation setting (Brooks et al., 2004) However, the use of VR in rehabilitation is not only useful as an assessment tool, but also as the potential to offer a training method restoring the memory function.

\section{COMPUTER-BASED COGNITIVE RETRAINING}

CBCR, based on intensive repetition, aims at improving cognitive skills needed to successfully receive sensory input, process information, and react without any use of external aids (Li et al., 2013). CBCR is available to the patient at home and offers stimulating tailored programs that can be modified to the individual's progress. Ample evidence is found suggesting CBCR is effective in the recovery of working memory (WM) (Olesen et al., 2004). Studies investigating CBCR in healthy participants showed that training can increase WM capacity and that training-induced changes in brain activity occur (Olesen et al., 2004; Westerberg et al., 2007). Additionally, training effects can be generalized to non-trained WM tasks, and to tests on attention, reasoning, and problem solving. Transfer of the training effects to non-trained WM tasks is consistent with the notion of training-induced plasticity in a common neural network for WM. The observed training effects suggest that WM training could be used as a remediation intervention for individuals for whom low WM capacity is a limiting factor in everyday life (Klingberg, 2010).

\section{NON-INVASIVE BRAIN STIMULATION}

Different neurophysiologic strategies to increase the activity of the injured brain area have been proposed mainly using Transcranial Magnetic Stimulation (TMS) and Transcranial Direct Current Stimulation (tDCS). TMS is based on the principle of electromagnetic induction and causes depolarization and hyperpolarization in the neurons. Lower frequencies of repetitive TMS is called repetitive Transcranial Magnetic Stimulation (rTMS), this 
is a train of TMS pulses delivered at constant intervals on the same intensity (low-frequency $1-4 \mathrm{~Hz}$, high-frequency $5-10 \mathrm{~Hz}$ ). rTMS presents the opportunity to interact even more effectively with cortical activity (Miniussi et al., 2008). Transcranial Direct Current Stimulation (tDCS) consists of placing 2 rubber electrodes on the scalp in order to allow a weak direct current to flow from anode to cathode. The electrical stimulus that reaches the brain is of enough intensity to modify the level of spontaneous neuronal excitability and activity by changing the resting membrane potential. tDCS is easier to apply and less expensive then TMS (Johansson, 2011).

Several studies emphasize the fact that interacting with cortical activity by cortical stimulation can positively affect cognitive performance and improve the rehabilitation potential (Miniussi et al., 2008). The therapeutic strategy of NBS consists of modulating an adaptive organization, allowing for the formation of functionally appropriate neural connections and enhancing behavioral recovery (Villamar et al., 2012). Preliminary evidence suggests that NBS may play a role in treating unilateral neglect (Nyffeler et al., 2009; Lim et al., 2010) and aphasia (Naeser et al., 2005; Szaflarski et al., 2011).

To summarize, the aim of this review is to provide an overview of the studies characterizing the most discussed memory remediation-oriented techniques developed in the last decade; VR, CBCR, and NBS.

\section{METHODS}

\section{SEARCH METHOD AND ARTICLE SELECTION}

A systematic literature search was performed using PubMed and Web of Science for studies published between January 2004 and August 2014 using the terms Acquired Brain Injury, (Traumatic) Brain Injury or Stroke in combination with Virtual Reality, Computer-based Cognitive Retraining, Computerized Training, Non-Invasive Brain Stimulation, Transcranial Magnetic Stimulation, repetitive Transcranial Magnetic Stimulation, or transcranial Direct Current Stimulation as well as Memory. The search in PubMed was limited in the following features: publication date (published in the last 10 years), species (human), adults ( $\geq 19$ years of age), and language (English). Likewise, the search in Web of Science was limited in the following features: language (English) and time span (2004 to 2014).

Intervention studies for improving memory function after ABI were selected when they met the following inclusion criteria: (1) individuals experiencing memory deficits resulting from $\mathrm{ABI}$ as confirmed by neurological examination; (2) $\geq 18$ years of age; (3) studies using specific measurements of memory functioning consisting of objective measures of memory function using standardized memory tests or batteries; and (4) had a study design with at least a pre and post intervention measurement. Memory treatment was considered any cognitive intervention attempting to improve memory, with neuropsychological tests as outcome measures. Studies published in languages other than English were excluded.

The first author (L.A.S.) conducted the search and screened the titles and abstracts, followed by an exclusion of duplicates. From screen-positive abstracts, full-text articles were collected when available and evaluated. In case the first author had doubt about inclusion of an article, the other authors were consulted. Articles meeting the aforesaid criteria were included in the final selection. The final selection was checked by the other authors as well.

\section{DATA EXTRACTION}

After the final selection, data extraction was performed by the first author (L.A.S.) and was based on data extraction methods from similar reviews (Schrijnemaekers et al., 2013). In case of doubt about which data to be extracted, the other authors were consulted. The following study characteristics were extracted from the articles: authors, design of the study, number of patients, outcome measures, $p$-value, and timing of measurements. The following intervention characteristics were extracted from the articles: aim of intervention, type of intervention, duration and intensity. The following patient characteristics were extracted from the articles: diagnostic criteria and severity, age, and time after onset. Results were considered to be positive when statistically significant at the $P<0.05$ level.

\section{QUALITY ASSESSMENT}

Two authors (L.A.S. and T.C.W.N.) independently appraised the characteristics and the quality of the selected studies. The methodological quality was evaluated based on the following elements: (1) randomization of intervention or different condition, (2) comparison of an experimental group and a control group that received either an alternative form of treatment or no memory intervention, (3) blinding of participants, (4) blinding of researchers, (5) reporting completeness of follow-up (Tijssen and Assendelft, 2003). We added 3 relevant elements to evaluate the methodological quality: (6) group size ( $\geq 10$ per group), (7) reporting effect size, and (8) reporting time post-ABI. We consider it important to report the time between the injury onset and the start of the intervention, to facilitate a comparison of the effect and to gain insights into the phase in which the patients were at time of the intervention (sub-acute phase vs. chronic phase). This 8-point checklist yielded a total score between 0 and 8 . Each study were giving a total score and consequently divided into high (total scores $\geq 6$ ), moderate ( $\geq 3$ and $\leq 6$ ), and low ( $\leq 3$ ) quality studies (Schrijnemaekers et al., 2013). Additionally, a distinction was made between effectiveness studies and feasibility studies for the interpretation of the results of each study.

\section{RESULTS}

The initial search identified 786 articles that were evaluated according to the inclusion criteria. Ultimately, 15 articles met the full inclusion criteria and were used for this review (see Figure 1). Of these 15 studies, 3 studies represented the VR technique, 7 studies represented CBCR, and 5 studies NBS. The specifics of the selected studies are presented in Tables 13 for results on VR, CBCR, and NBS respectively. After briefly describing these studies, we present the findings of the methodological quality based on the elements mentioned above (for total overview see Table 4). There was a 95\% agreement between the 2 authors (L.A.S. and T.C.W.N.) regarding the quality assessment. 


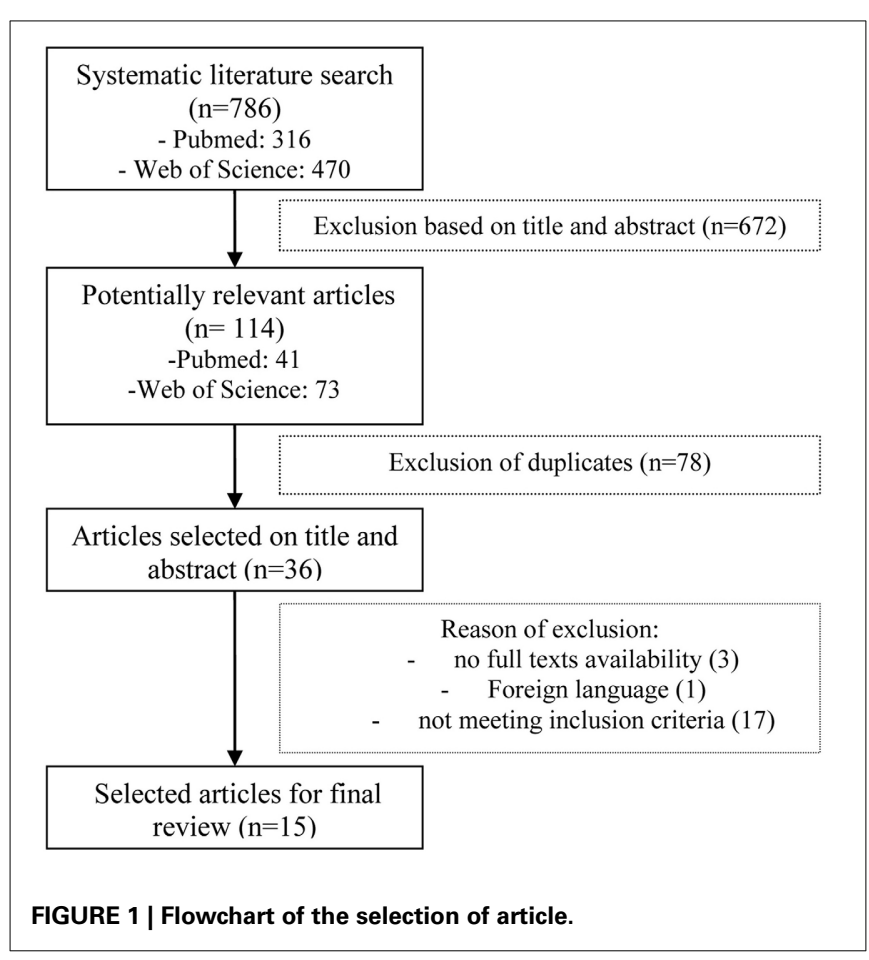

\section{VIRTUAL REALITY}

A small-sample $(n=4)$, pre and post experimental design was developed to initially study the usability and efficacy of a VRbased training program for people with $\mathrm{ABI}$ (Yip and Man, 2009). Outcome measures were at the level of memory function. A VR-based community living skills training of 10 sessions were given, consisting of key cognitive training elements (including memory) to promote generalization to real-life situations. Measurements consisted of built-in parameters to document the participants' performance during each session and the Neurobehavioral Cognitive Status Examination. Due to the explorative and qualitative character of this study, a statistical significance was not established. However, a positive training effect was shown by the outcome measures and narratively presented. All 4 patients showed improvement in skills acquisition on the community living tasks and in memory performance on neuropsychological measurements. All patients showed the same improvement in performing the tasks when tested again in a real-life environment.

Caglio et al. (2012) described a qualitative case-study of a 24-year-old man with TBI presenting memory deficits and evaluated the efficacy of a 3D interactive VR navigational training program measuring neuropsychological changes and fMRI modification cerebral activations. Measurements consisted of a functional neuroimaging assessment and a standardized neuropsychological assessment on frontal executive functions, general cognitive functions, and various memory functions (i.e., spatial short-term memory, visual-spatial learning, WM, verbal learning). Visual-spatial memory improvement appeared to be present both after the VR navigational training and in followup testing. The functional neuroimaging assessment showed an increased cerebral activity in the left hippocampus and the right parahippocampal cortex compared to the pre-training assessment.

Four years later, Yip and Man described the effectiveness of a VR-based memory training in a larger sample $(n=37)$ (2013). Using a Randomized Controlled Trial (RCT), the effectiveness was evaluated of a VR-based cognitive rehabilitation program using prospective memory as training content. While the experimental group received a 12-session VR-based program, the control group did not received any VR-based training, but did attend regular readings and table game activities during the treatment phase. Neuropsychological tests were administered to measure the effects of the treatment on prospective memory skill acquisition (VRbased assessment by outcome parameters), prospective memory, learning, and executive function. The results showed significantly larger changes in both VR-based and real-life prospective memory outcome measures after the training. Related cognitive attributes such as frontal lobe functions and semantic fluency showed a significant improvement compared with the control group. A significant improvement is found in the real-life behavioral assessment in prospective memory due to the training, indicating a transfer of learnt skills in a virtual environment to a real-life setting.

The study of Yip and Man (2013) was considered to be of high quality. Both the studies of Caglio et al. (2012) and Yip and Man (2009) were considered to be of low quality based on the quality assessment. It should be noted however that a true comparison was difficult to make as 2 studies were qualitative research (Yip and Man, 2009; Caglio et al., 2012) whereas 1 was quantitative research (Yip and Man, 2013).

To sum up, although the 3 studies do identify an improvement in memory function after VR-based training, it is difficult to draw any conclusions as the number of articles available was limited. Besides, of all the available articles available only 1 study was considered of high quality.

\section{COMPUTER-BASED COGNITIVE RETRAINING}

Using a RCT, the effects of intense adaptive WM training in stroke patients were investigated (Westerberg et al., 2007). A sample of 18 patients was randomly divided into an experimental or passive control group. The experimental group was trained using computer-based visuo-spatial and auditory WM tasks at home. The training method was implemented with the software program Cogmed QM (Cognitive Medical Systems). The control group only performed the neuropsychological test battery with no training in between at baseline and after 5 weeks. Both WM and attention abilities improved significantly within the experimental group, but not within the passive control group.

In a cross-over RCT, the short- and long-term transfer effects of a computerized WM training program were evaluated for patients suffering of WM deficits after ABI (Lundqvist et al., 2010). A sample of 21 patients was randomly divided into 2 groups. The experimental group received systematic WM training (Cogmed QM), whereas the control group did not receive any training during the same period. The patients were assessed at baseline, after 4 and 20 weeks with neuropsychological tests focusing on verbal and visual WM. There was a significant improvement on the trained WM tasks and the non-trained WM tasks 


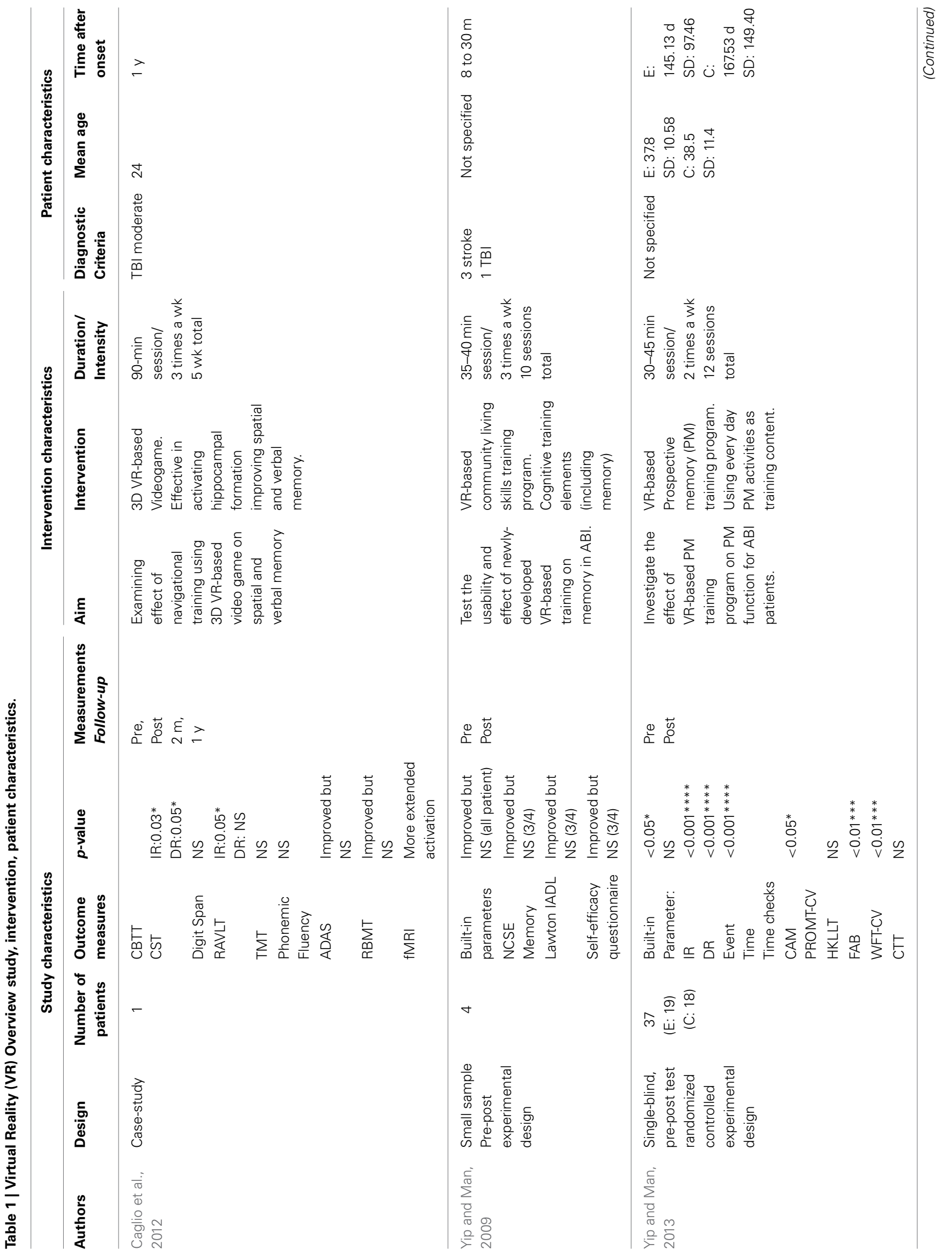




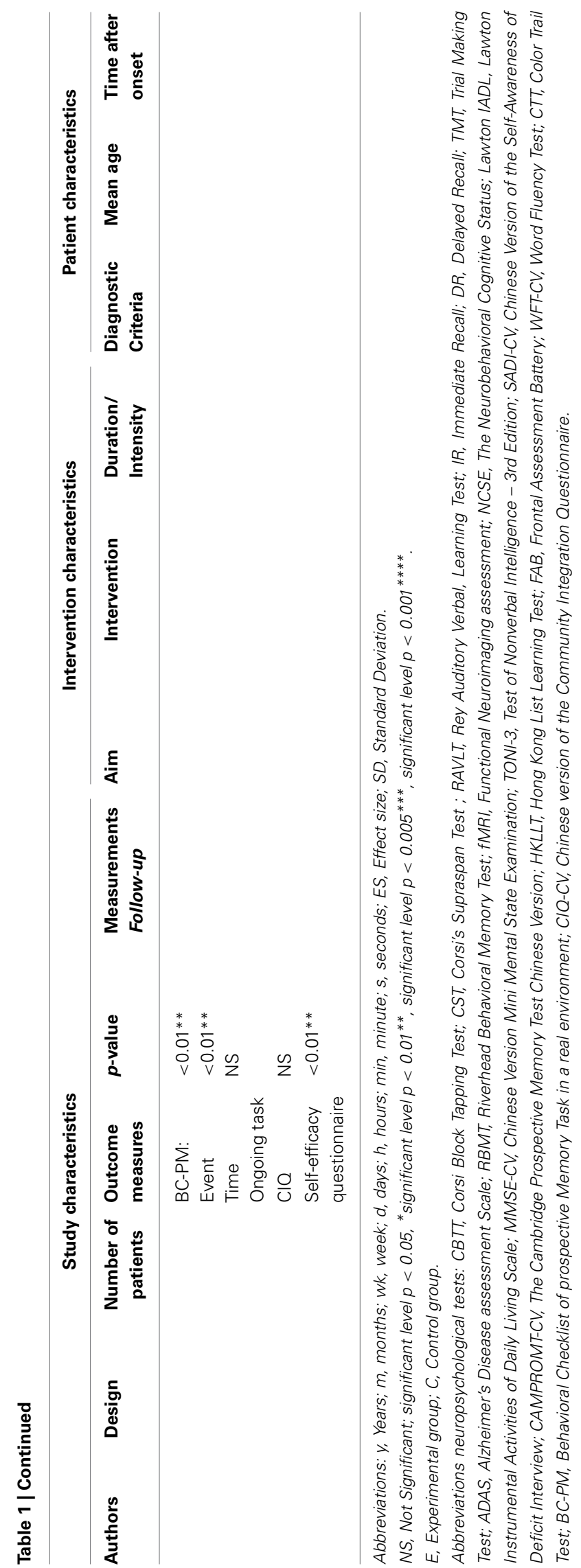

as measured by neuropsychological tasks at 4 and 20 weeks after training compared to baseline.

A prospective cohort study evaluated the effectiveness of a computerized training using Cogmed QM software (Johansson and Tornmalm, 2012). A sample of $18 \mathrm{ABI}$ patients attended the training 3 times a week. The patients were assessed before, during, after the WM training, and additionally at a 6 months followup with WM assessments. The computerized training showed a significant improvement on trained WM tasks. The effect was maintained at the 6 month follow-up. The study supports the idea that a computerized WM training program can affect WM functioning for ABI patients.

An additional RCT assessed the effectiveness of computerized WM training (Cogmed QM) on WM functioning in ABI patients (Björkdahl et al., 2013). A sample of 38 ABI patients were randomly assigned to an experimental group or control group and received 5 weeks of standard rehabilitation in accordance with the usual routine at the clinic. The experimental group was offered an additional training with the Cogmed QM training program. To explore the impact of the training, assessments were done at baseline, after the training program, and at a follow-up 3 months later. The assessment consisted of neuropsychological tests and a WM questionnaire measuring WM on functional level (i.e., level of activity in daily life). The experimental group improved significantly more compared to the control group. Cogmed QM showed a generalized effect on non-trained WM tasks.

Using a quasi-experimental pre-test and post-test design the effectiveness of a CBCR program was evaluated on improving memory and attention deficits for adults with $\mathrm{ABI}$ ( $\mathrm{Li}$ et al., 2013). A sample of 12 patients was assessed using the Cognistat Assessment as pre-test and post-test measurement. Each patient completed 8 sessions using the attention and memory subprograms of the Parrot Software, which is an interactive rehabilitation program with over 100 subprograms designed to improve cognitive function. Significant improvement was found in both memory and attention measured by the Cognistat Assessment scores.

A RCT investigated whether patients with a dysfunctional WM could improve their WM, cognitive function, and psychological health using a computerized WM training with the Cogmed QM program (Akerlund et al., 2013). A sample of 47 patients, in the sub-acute phase after $\mathrm{ABI}$, were randomly assigned into an intervention group and a control group. Various WM neuropsychological tests were administered at baseline, post-intervention, and at a follow-up of 18 weeks. Both groups underwent integrated rehabilitation. The intervention group also attended the computerized WM training program, which was offered to the control group after the completion of the study. Both the Barrow Neurological Institute Screen for Higher Cerebral Functions (BNIS) and the Digit Span differed significantly between the intervention and control group due to the greater improvement in the intervention group after the WM training. Both groups improved after WM training on the BNIS, the Digit Span, and the WAIS III WM scale. Additionally, psychological health improved as both groups reported less depressive symptoms. 


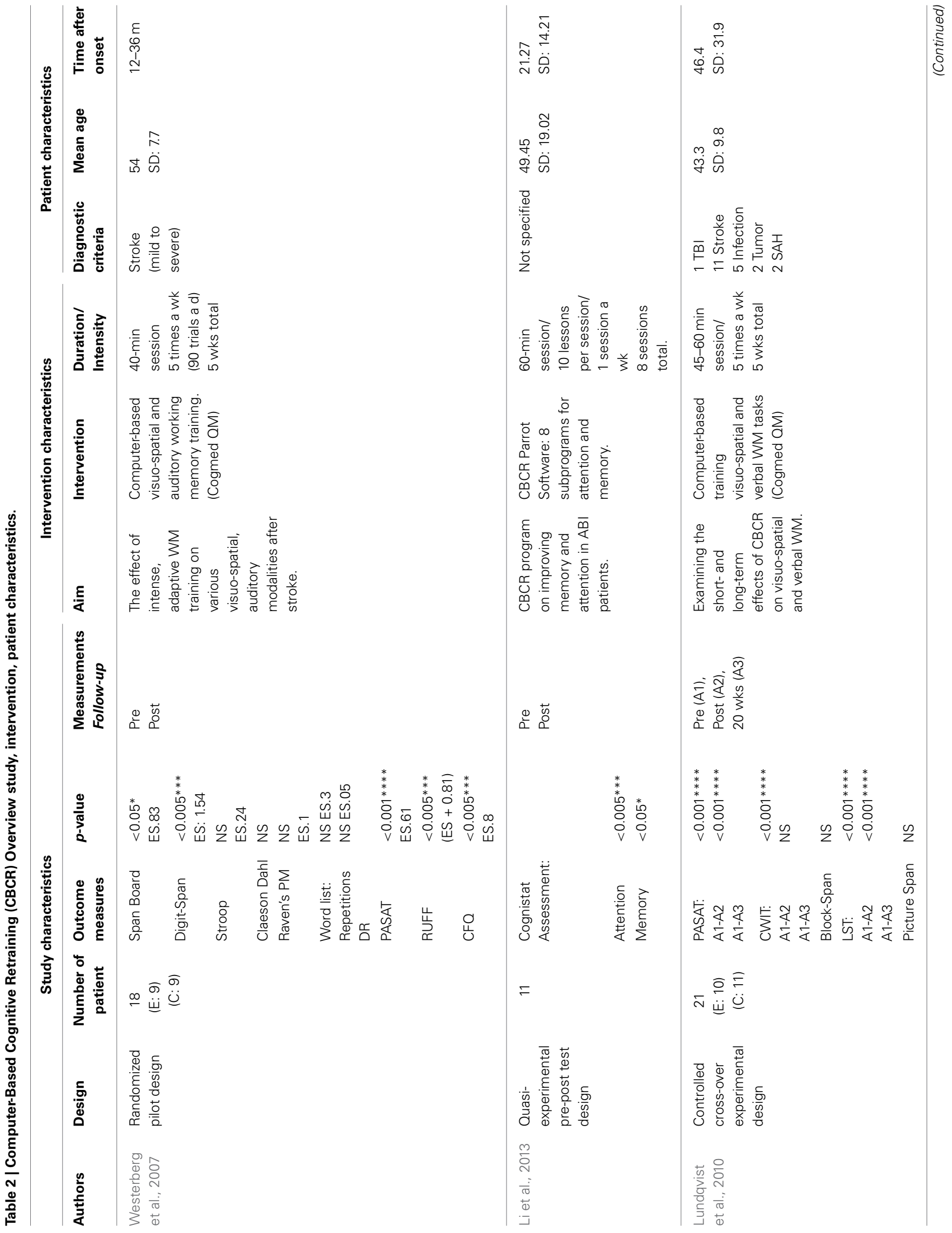




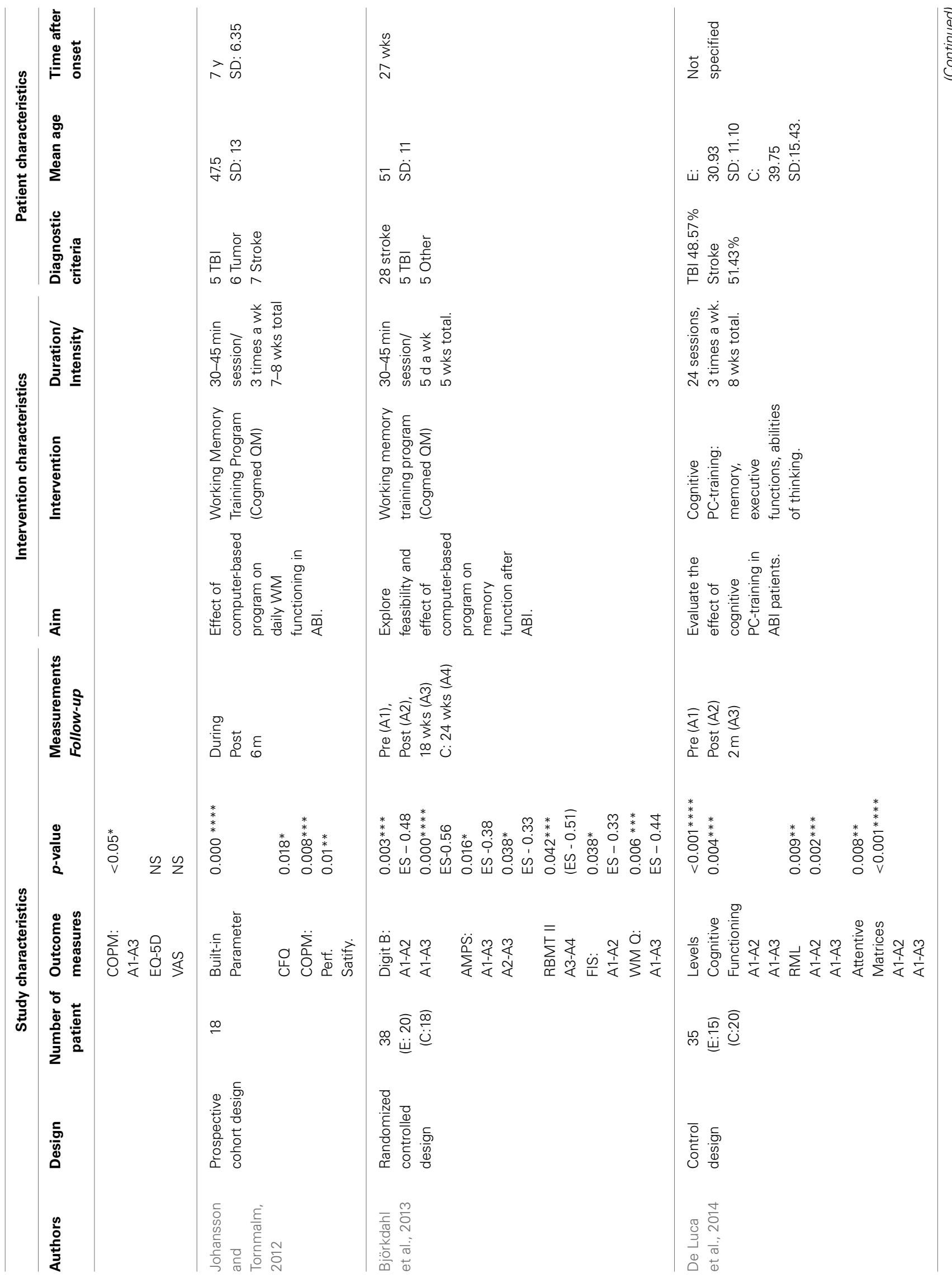




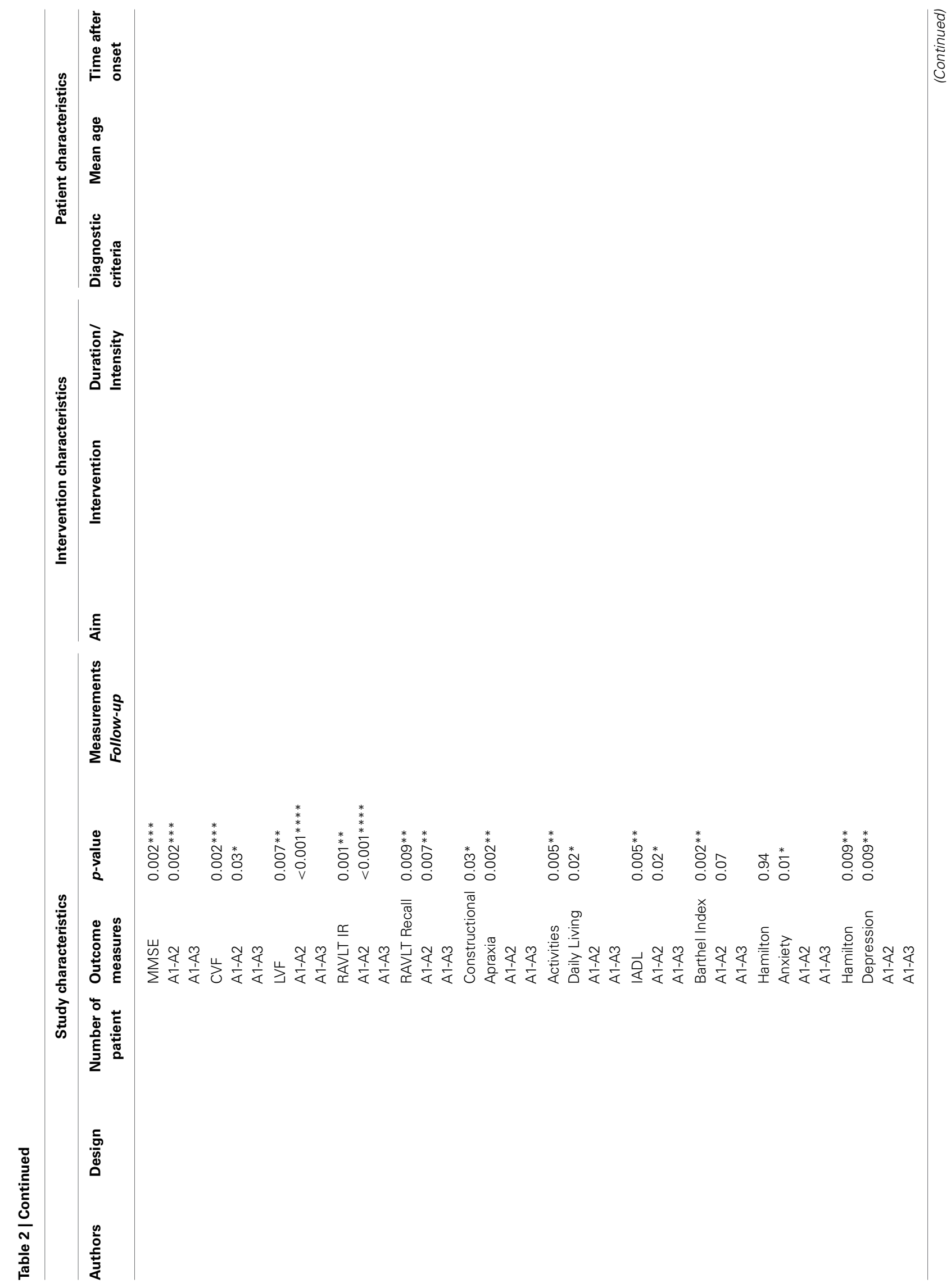




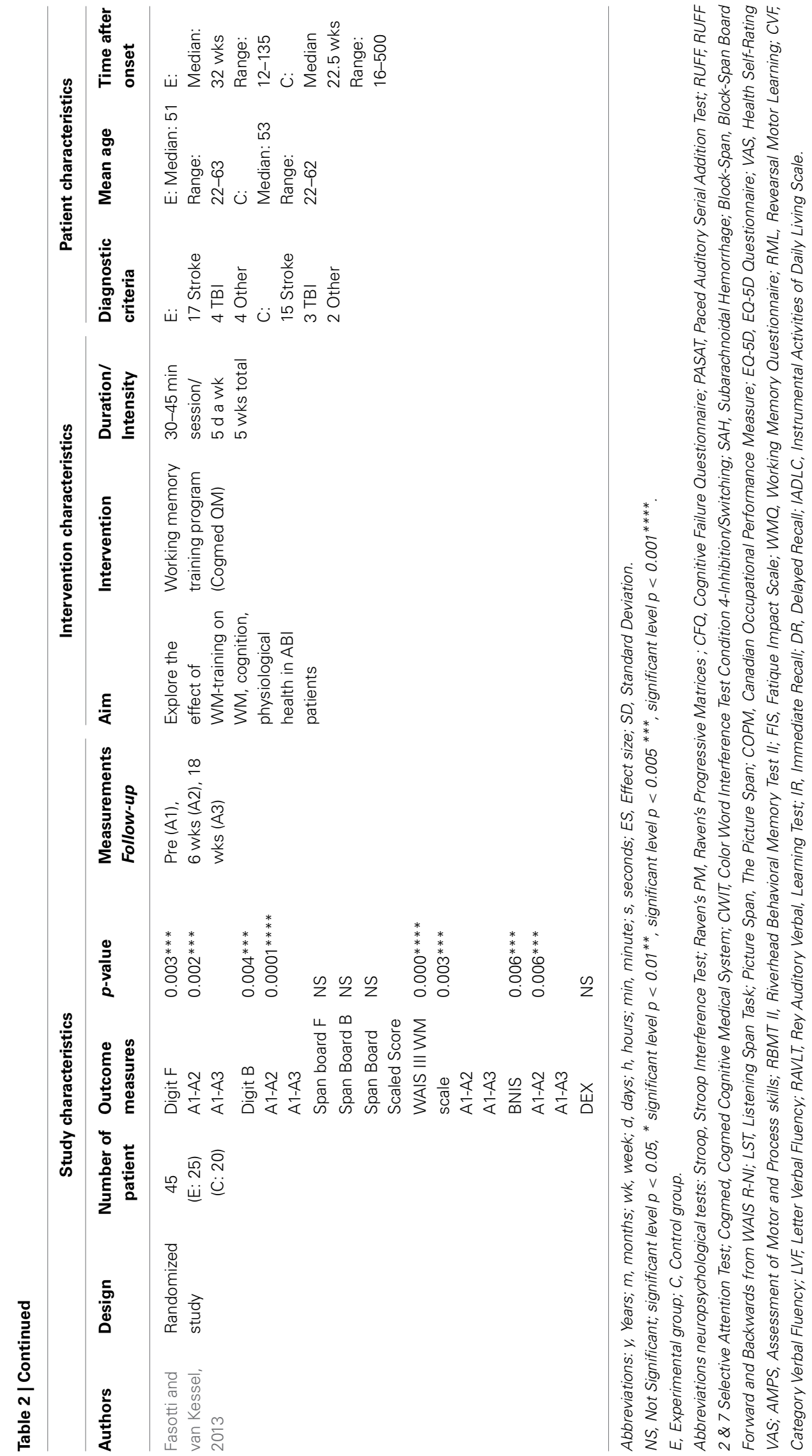




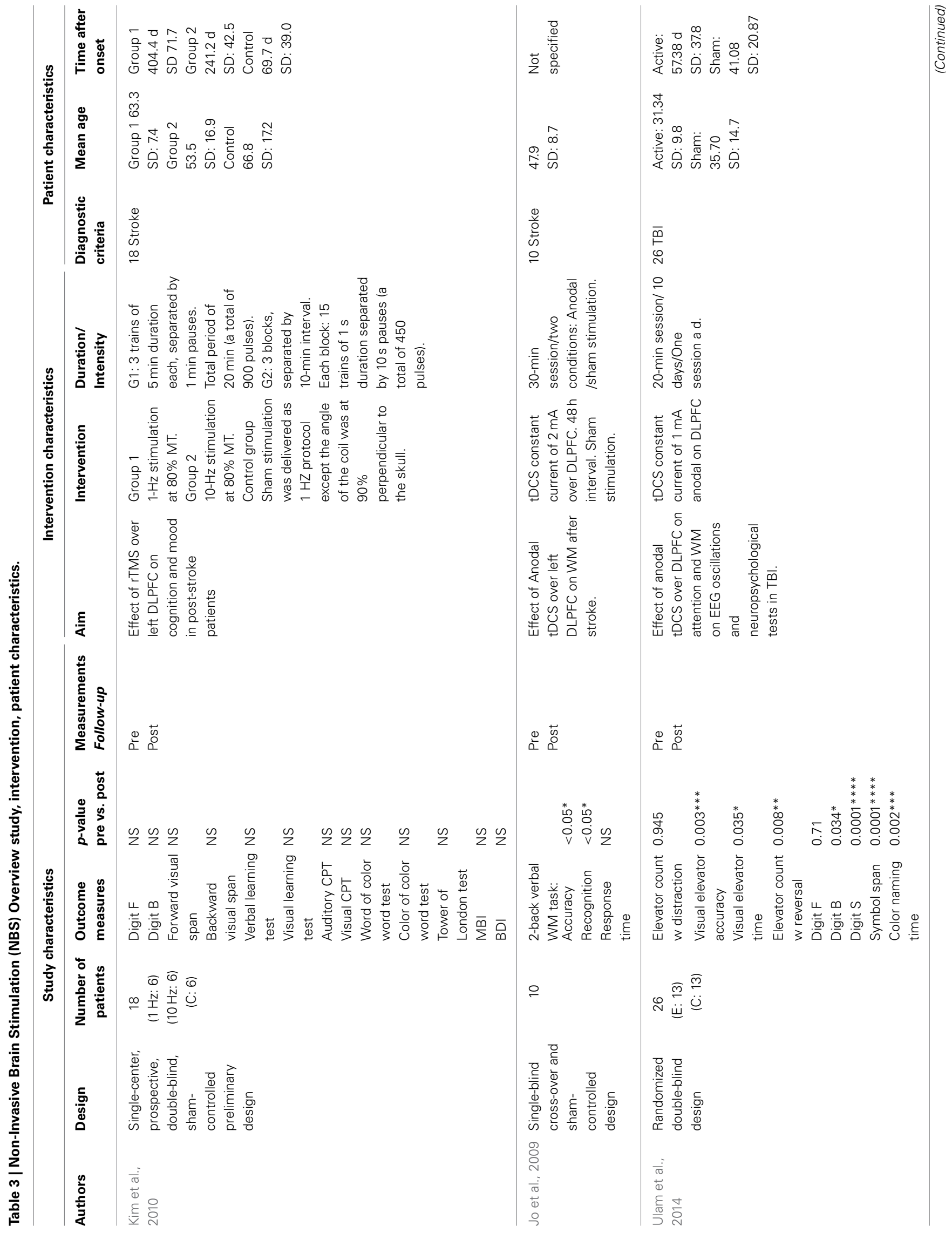




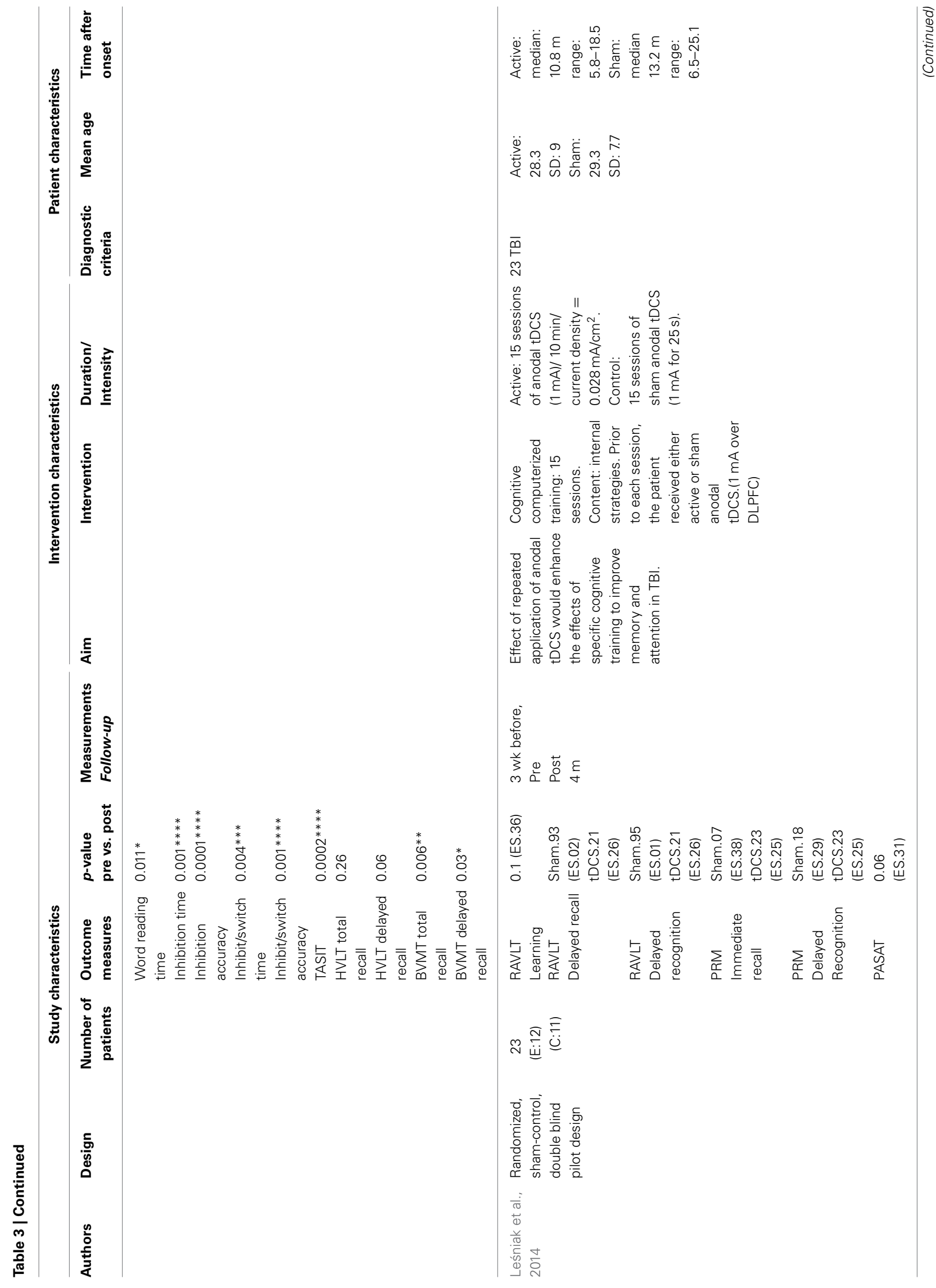




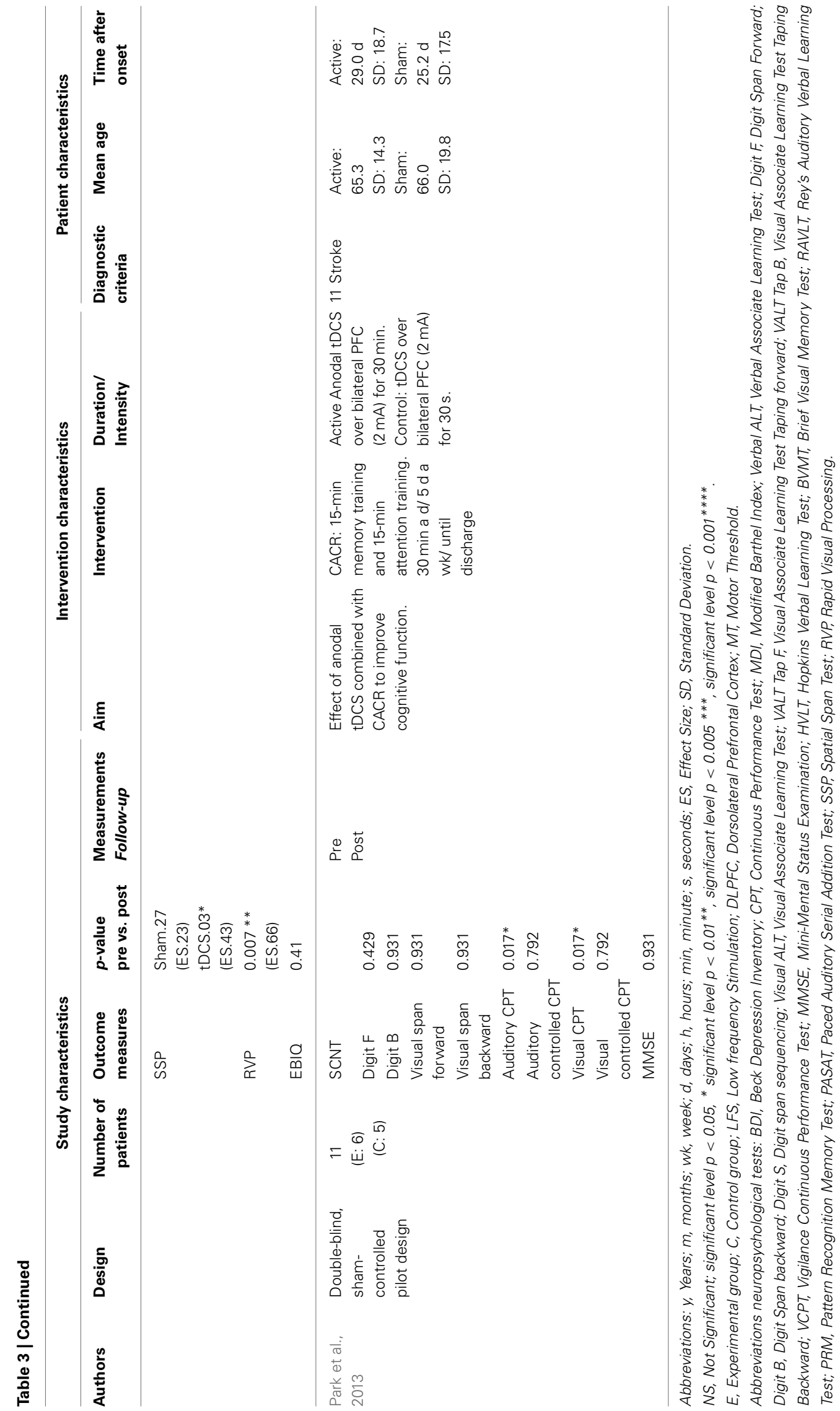


Table 4 | Scores of the quality assessment of the discussed studies, based on 8 elements.

\begin{tabular}{|c|c|c|c|c|c|c|c|c|c|c|c|}
\hline Study & 1 & 2 & 3 & 4 & 5 & 6 & 7 & 8 & Total score & Quality & Aim \\
\hline \multicolumn{12}{|l|}{ VR } \\
\hline Caglio et al., 2012 & 0 & 0 & 0 & 0 & 1 & 0 & 0 & 1 & 2 & Low & Feasibility \\
\hline Yip and Man, 2013 & 1 & 1 & 0 & 1 & 1 & 1 & 0 & 1 & 6 & High & Effectiveness \\
\hline \multicolumn{12}{|l|}{ CBCR } \\
\hline Lundqvist et al., 2010 & 1 & 1 & 0 & 0 & 1 & 1 & 1 & 1 & 6 & High & Effectiveness \\
\hline Johansson and Tornmalm, 2012 & 0 & 0 & 0 & 0 & 1 & 1 & 0 & 1 & 3 & Moderate & Feasibility \\
\hline Björkdahl et al., 2013 & 1 & 1 & 0 & 0 & 1 & 1 & 1 & 1 & 6 & High & Effectiveness \\
\hline De Luca et al., 2014 & 1 & 1 & 0 & 0 & 1 & 1 & 0 & 1 & 5 & Moderate & Effectiveness \\
\hline Åkerlund et al., 2013 & 1 & 1 & 0 & 0 & 1 & 1 & 0 & 1 & 5 & Moderate & Effectiveness \\
\hline Leśniak et al., 2014 & 0 & 1 & 1 & 1 & 1 & 1 & 1 & 1 & 7 & High & Effectiveness \\
\hline Park et al., 2013 & 1 & 1 & 1 & 1 & 0 & 0 & 0 & 1 & 5 & Moderate & Effectiveness \\
\hline
\end{tabular}

0, Negative; 1, Positive.

High was considered total scores $\geq 6$, moderate $\geq 3$ and $\leq 6$, and low $\leq 3$.

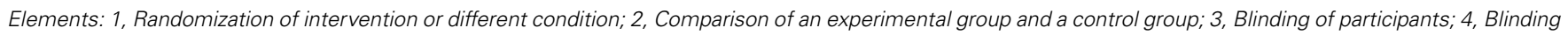
of researchers; 5, Reporting completeness of follow-up; 6, Group size $\geq 10$ per group; 7, Reporting Effect Size; 8, Reporting time post-ABI.

A RCT evaluated the effects of a cognitive pc-training with regard to semantic memory, verbal fluency, and short-term auditory-verbal memory in ABI patients (De Luca et al., 2014). A sample of $35 \mathrm{ABI}$ patients were randomly divided into 2 groups. Cognitive impairment was investigated through the use of a psychometric battery, administered before and 2 months after the cognitive pc-training. The cognitive pc-training was performed only by the experimental group, in addition to conventional treatment. After the training, the results showed a global improvement in both of the groups. However, the experimental group showed a greater cognitive improvement than the control group, with significant differences in all the neuropsychological tests performed. The results suggest that cognitive pc-training may be a promising methodology to optimize rehabilitation outcomes following ABI.

Based on the evaluated elements from the quality assessment, the studies of Westerberg et al. (2007), Lundqvist et al. (2010), and Björkdahl et al. (2013) were considered of high quality (see Table 4). The studies of Johansson and Tornmalm (2012), De Luca et al. (2014), and Akerlund et al. (2013) were considered of moderate quality. The study of Li et al. (2013) was considered of low quality.

To sum up, the 7 studies reported a significant improvement of the memory function after the completion of CBCR. As 6 studies were considered of moderate/high quality, these findings support the idea that $\mathrm{CBCR}$ may be a promising methodology to optimize the recovery of the memory function in $\mathrm{ABI}$ patients.

\section{NON-INVASIVE BRAIN STIMULATION}

A single-blind, cross-over, and sham-controlled study investigated whether anodal tDCS over the left dosolateral prefrontal cortex would affect the WM performance of post-stroke patients (Jo et al., 2009). A sample of 10 patients participated in 2 stimulation conditions (anodal stimulation with a constant current of $2 \mathrm{~mA}$ and sham stimulation). The order of stimulation was randomly assigned. Each stimulation session was separated by at least $48 \mathrm{~h}$ to wash out the effects of the previous run. All patients performed a two-back WM task before and after the administration of the tDCS. A significant improvement in accuracy and recognition accuracy was only found in the anodal tDCS and not in the sham tDCS. Anodal tDCS applied over the left dorsolateral prefrontal cortex at an intensity of $2 \mathrm{~mA}$ was associated with enhanced verbal WM performance in patients after stroke.

A double-blind RCT examined whether rTMS applied over the left dorsolateral prefrontal cortex affected cognition and mood in post-stroke patients (Kim et al., 2010). A sample of 18 patients were enrolled and randomly assigned to 1 of 3 treatment groups: low-frequency $(1 \mathrm{~Hz})$ stimulation, high-frequency $(10 \mathrm{~Hz})$ stimulation, and sham stimulation (control). Each patient underwent 10 consecutive treatment sessions ( 5 times a week for 2 weeks). A complete neuropsychological battery was performed to evaluate various domains of cognition such as verbal and visual memory, executive functioning, attention, working memory, and visuomotor coordination. The Beck Depression Inventory was used to assess mood status. These assessments were conducted in all patients before and after treatment. Treatment had no significant effect on any cognitive function parameter in any of the 3 groups. In contrast, high-frequency rTMS resulted in significantly lower Beck Depression Inventory scores compared with baseline, and compared with the other 2 groups. These preliminary data 
suggests that there was a positive effect on mood, but the study was not powered to detect any measurable effect on cognition including memory.

A double-blind RCT investigated the synergistic effects of both computer-assisted cognitive rehabilitation (CACR) and tDCS on cognitive function (attention and memory) in post-stroke patients (Park et al., 2013). A total of 11 patients were randomly divided into an active tDCS group and a control group. Both groups received CACR training for $30 \mathrm{~min}$ a day $(15 \mathrm{~min}$ memory training, 15 min attention training) 5 times a week until discharge. The tDCS group completed the CACR program during a mean period of 18.5 days combined with the anodal tDCS (over the bilateral prefrontal cortex). The control group also completed the CACR program (mean period of 17.8 days) combined with tDCS, except that the current was reduced to 0 after $30 \mathrm{~s}$. All patients were evaluated using the Korean Mini-Mental State Examination and the Seoul Computerized Neuropsychological Test (SCNT). The SCNT was composed of 10 measurements, assessing the verbal memory, visuospatial memory, attention, and visuo-motor coordination. The patients of the tDCS group showed a significant improvement in 2 attention tests of the SCNT items. The results indicated that the combined use of tDCS and a CACR program may provide beneficial effects in improving attention. However, no evidence was found for the memory function.

A double-blind RCT investigated the cumulative effects of anodal tDCS on EEG oscillations, attention, and WM function among patients with TBI (Ulam et al., 2014). A sample of 26 patients were randomly assigned to active or sham tDCS groups. EEGs were recorded at 6 different time points, assessing both immediate and cumulative effects of tDCS on EEG oscillations. Twenty-minutes sessions of $1 \mathrm{~mA}$ anodal tDCS over the left dorsolateral prefrontal cortex were provided on 10 consecutive days for the active group. For the sham tDCS group, current gradually faded in over a period of $8 \mathrm{~s}$, followed by $30 \mathrm{~s}$ of stimulation, with the current then fading out over an additional $8 \mathrm{~s}$. Neuropsychological tests were administered before and after the series of tDCS sessions. While attention and WM was the primary interest, other outcome measures were included. Results show that no between-group differences were present for any of the tests administered. Both the active tDCS and sham tDCS showed an equal number of statistically significant improvements (15 out of 19 tests). The EEG revealed immediate and cumulative changes in brain oscillations for the active tDCS, but not in the sham group. Results suggest that 10 anodal tDCS sessions may beneficially modulate regulation of cortical excitability for patients with TBI. However, tDCS does not show greater improvements on neuropsychological test (including measurements of WM and attention) compared to sham tDCS.

A double-blind RCT determined whether cumulative anodal tDCS over the left dorsolateral prefrontal cortex could enhance rehabilitation of memory and attention in patients with TBI (Leśniak et al., 2014). A sample of 23 patients were randomly assigned to 2 groups. The experimental group received anodal tDCS ( $10 \mathrm{~min}$ of $1 \mathrm{~mA}$ ) on a daily basis for 15 days followed by rehabilitative cognitive training. The control group received anodal tDCS in the first $25 \mathrm{~s}$ of a $10 \mathrm{~min}$ stimulation period (sham condition) with the same rehabilitation. A battery of memory and attention neuropsychological tests were administered which included visual and auditory modalities. Participants were tested twice before the intervention (to control for spontaneous recovery), after the intervention, and 4 months later. After treatment the experimental group exhibited larger effect sizes in 6 of $8 \mathrm{cog}$ nitive outcome measures, but they were not significantly different from controls. At follow-up, differences remained insignificant. This study did not provide sufficient evidence to support the efficacy of repeated anodal tDCS for enhancing rehabilitation of memory and attention in patients after severe TBI.

Based on the quality assessment the studies of Ulam et al. (2014) and Leśniak et al. (2014) were considered of high quality. The studies of Jo et al. (2009), Kim et al. (2010), and Park et al. (2013) were considered of moderate quality.

To sum up, only 1 study (Jo et al., 2009) detected a significant enhanced verbal WM performance after the use of NBS in stroke patients. Four studies (Kim et al., 2010; Park et al., 2013; Leśniak et al., 2014; Ulam et al., 2014) do not find sufficient evidence to support the efficacy of NBS for enhancing rehabilitation of memory in $\mathrm{ABI}$ patients.

\section{DISCUSSION}

The aim of this systematic review was to describe memory rehabilitation interventions based on remediation-oriented techniques post $A B I$ and evaluate its effectiveness. We found 15 studies in the last 7 years (2007-2014), evaluating 3 memory remediation approaches; 3 studies on VR, 7 on $\mathrm{CBCR}$ and 5 on NBS. Considering the quality of the studies, only 1 study representing VR completed the requirements of a RCT, 3 studies representing the NBS technique fulfilled the RCT requirements, whereas 5 studies representing CBCR consisted of a RCT design. It appeared, based on the quality assessment that CBCR was the most promising as the methodological quality was high. Importantly, CBCR is found effective in improving the memory function post ABI. Although the VR studies did find a positive effect, there was only a low number of studies available and the quality of the studies was also considered low. Four of the 5 studies evaluating NBS did not find significant improvement of the memory function and the quality of these studies were considered moderate to high. Only 1 of the studies evaluating NBS did find positive results, yet the quality of this study was considered moderate. More details of the findings will be discussed below for each technique separately.

\section{COMPUTER BASED COGNITIVE REHABILITATION}

All 7 studies have shown that CBCR can be effective in improving memory function in individuals with $\mathrm{ABI}$ (Westerberg et al., 2007; Lundqvist et al., 2010; Johansson and Tornmalm, 2012; Åkerlund et al., 2013; Björkdahl et al., 2013; Li et al., 2013; De Luca et al., 2014). These findings are consistent with Klingberg's prediction (2010), suggesting that WM training could be used as a remediation-oriented intervention for individuals for whom low WM capacity is a limiting factor in everyday life.

Five studies (Westerberg et al., 2007; Lundqvist et al., 2010; Johansson and Tornmalm, 2012; Björkdahl et al., 2013; De Luca et al., 2014) investigated the generalized effect on functional level 
(i.e., level of activity in daily life) and found a positive effect. This was measured subjectively, using self-report questionnaires regarding daily activities relying on the memory function. This is interesting from a transfer-of-training point of view. Five studies measured the long-term effects, ranging from 18 to 24 weeks postintervention and found positive results (Lundqvist et al., 2010; Johansson and Tornmalm, 2012; Åkerlund et al., 2013; Björkdahl et al., 2013; De Luca et al., 2014).

Five studies randomly assigned their participants to an experimental vs. a control group and therefore completed the requirements of a RCT. Only 1 study used a double blind design (both patient and researcher were blind to the treatment of the patient) (Westerberg et al., 2007). Future research should focus on the establishment of several other criteria such as blinding of participants and researchers to strengthen the evidence.

In conclusion, we consider CBCR as most promising due to the positive results and the relatively high methodological quality of the selected studies. However, before proposing CBCR as rehabilitation intervention in clinical practice important criteria should be fulfilled. Future research should further define the effect of the intervention generalized to functional level, participation in society level, as well as the long-term effects and the effect on quality of life. Additionally, RCT's are needed focusing on several methodological criteria to strengthen evidence.

\section{VIRTUAL REALITY}

All 3 studies found a significant improvement of the memory function after a VR-based training (Yip and Man, 2009, 2013; Caglio et al., 2012). These positive findings are consistent with findings in healthy elderly participants experiencing memory deficits (Optale et al., 2009). This suggests that VR-based training could possibly be a valid part at encouraging memory recovery for individuals experiencing memory deficits.

Two studies also found a generalization of the effect in a real environment (Yip and Man, 2009, 2013). Patients showed the same improvement in performing the tasks when tested again in the real environment. As such, VR-based training seems to be able to retrain the underlying function in a virtual environment and facilitate the generalization to real-life performance.

No study investigated the effect of the training on functional level. Long term effects at 2 months and 1 year post-intervention were established in the study of Caglio et al. (2012), but should be interpreted with caution due to the low methodological quality (total score: 2). Both studies of Yip and Man (2009, 2013) have shown positive effect but for a limited time-window, namely only directly post-intervention as no follow up was performed at a longer interval post-training. In future studies, it would be desirable to extend the outcome measurements to establish the effect on functional level and the long-term effects. This could provide valuable information for clinical use.

It is important to note that the quality of the studies representing VR was low to moderate according to our quality assessment. Only 1 study (Yip and Man, 2013) met the requirements of a RCT and used a single blind design (blinding the researcher for the treatment). Two studies failed to apply a control group in their methodology. As a result, those studies did not blinded their participants nor their researchers. Future research should involve true replications, taking into account essential criteria such as randomization and the use of control group to obtain higher methodological quality.

In conclusion, despite the positive findings these results should be considered preliminary because of the limited number of studies available and the low number of ABI patients. The significant improvement on memory performance for $\mathrm{ABI}$ patients is encouraging. However, insufficient evidence is available to be proposed as treatment in clinical practice.

\section{NON-INVASIVE BRAIN STIMULATION}

Four studies did not detect significant improvement in the memory function after the use of NBS (Kim et al., 2010; Park et al., 2013; Leśniak et al., 2014; Ulam et al., 2014). According to the quality assessment these studies were considered moderate to high (total score ranging from 4 to 7 ). Only 1 study found a significant WM improvement (Jo et al., 2009), however the methodological quality of this study was considered moderate (total score: 4).

These disappointing findings were unexpected due to the promising findings in healthy participants. Several studies did find significant improvement in memory tasks due to NBS in healthy participants (Kessels et al., 2000; Oliveri et al., 2001; Luber et al., 2007; Preston et al., 2010). A recent review detected positive effects of rTMS and tDCS improving measures of WM performance, including reaction time and/or accuracy (Brunoni and Vanderhasselt, 2014). These results were only found when the NBS was applied over the dorsolateral prefrontal cortex (DLPFC) (Brunoni and Vanderhasselt, 2014). On the contrary, 3 studies of the present review did not detect significant improvement of the memory function, even when NBS was applied over DLPFC (Kim et al., 2010; Leśniak et al., 2014; Ulam et al., 2014). Additionally, positive results were found in healthy elderly adults, whereas a significant improvement was found in accuracy of a verbal WM task due to anodal tDCS as compared to sham tDCS (Park et al., 2014). Unfortunately, the selected studies of this review did not find any of these findings in the ABI population except for 1 study (Jo et al., 2009). On the other hand, it is important to note that a healthy or an aging brain could possibly react very differently compared to the restoration mechanism of a damaged brain post ABI.

To summarize, on the basis of this review CBCR is considered the most promising novel approach of the last decade in view of the positive results and the high methodological quality of the studies. The number of studies representing VR was limited and the methodological quality low, therefore the results should be considered preliminary. The studies representing NBS did not find evidence that the use of NBS could improve memory function and these studies were considered of moderate to high quality. Therefore, on the basis of the knowledge available we recommend CBCR as promising remediation-oriented intervention to improve memory function post ABI.

This review stresses some important limitations of the literature available on remediation-oriented memory interventions after ABI. First, the ability to benefit of those techniques may vary depending on what kind of injury the individual suffers from Fish et al. (2008). This review focused on memory impairment 
in a heterogeneous $\mathrm{ABI}$ patient population group with different injury-related diagnoses. This could be considered as a limitation, as each brain injury has a different pathology (e.g., focal vs. diffuse) and different demographics (e.g., age), which result in different restoration mechanisms such as different time courses and magnitudes of recovery. TBI is associated with a hallmark pattern of pathology concerning direct damage to frontal and temporal lobes, plus diffuse axonal injury resulting from tearing and shearing mechanisms (Levine et al., 2006). This causes a reduction in gray and white matter and impairs connectivity. The focal damage resulting from stroke is more diverse. In addition to a difference in pathology, the demographics of TBI and stroke are divergent. Stroke primarily affects people over 65 years of age, whereas TBI incidence is highest in the 15-24 age groups. This difference in pathology and demographics result in different restoration mechanism. It would therefore have been preferable to make a distinction between TBI and stroke. However, we believe that selecting the ABI population for this review gave the possibility to collect a wider range of knowledge.

Second, sample size is a crucial issue in quantitative research which seeks to make statistically based generalizations from the study results to the wider ABI population. The sample sizes used in the available literature may be considered too small to draw firm conclusions. As well as the regular absence of a control condition, the lack of blinding of participants and researchers, and the explorative character of several studies. These limitations restricted the reliability of the study's conclusions and consequently restricted the ability for us to draw well-founded conclusions.

A major strength of our review is the inclusion of 15 studies that had not been evaluated by previous reviews. On the other hand a limitation of this review may be the selection of appropriate search terms. We only searched on the terms Acquired Brain Injury, (Traumatic) Brain Injury, or Stroke. This selection of search terms may be quite limited, as ABI is a collective term for many more injury-related diagnoses. The collective term ABI can be subdivided into two categories: traumatic brain injury (TBI; i.e., external force traumatically injures the brain due to accidents, assaults or neurosurgery) or non-traumatic injury derived from either an internal source (NTBI; stroke, brain tumor) or an external source (e.g., poisoning, substance abuse). The selected search terms may have failed to cover the complete $\mathrm{ABI}$ population, even if the majority of ABI is caused by TBI or stroke. It might therefore be possible that we missed relevant studies.

A second limitation of this review may be the selection of the inclusion criteria. Solely studies evaluating interventions, with the focus primarily on improving the memory function were selected. Consequently, several studies using neuropsychological memory assessments as included outcome measure were excluded, since the primary outcome measure was variant (e.g., depression). These studies were excluded as they did not met our inclusion criteria, although their findings might have been possibly relevant to our review.

A final limitation may be the fact that we did not focus on pharmacological interventions, even though medication could be considered a remediation-oriented intervention. The included pharmacological interventions could have had favorable and interesting effects on the memory function. Hence, this should be considered in future research. However, in case of this particular review, we feel that pharmacological therapies were not suitable for targeting only the memory function without affecting other cognitive function.

In conclusion, the research on remediation-oriented interventions reviewed in this study represents just the beginning of a new research field that explores innovative possibilities for enhancing memory function in ABI patients. Even though CBCR in particular shows great promises, more research is needed to establish this remediation-oriented program as standard intervention in clinical practice, especially given the heterogeneity of $\mathrm{ABI}$, time course of spontaneous recovery, timing of training after $\mathrm{ABI}$, and generalization of effects at several levels of functioning. Although replication studies may seem less appealing, they are sorely needed in this field where many topics are novel and risk to remain novel (Fasotti and van Kessel, 2013).

\section{AUTHOR CONTRIBUTIONS}

Conception and design of the work: Lauriane A. Spreij, Johanna M. A. Visser-Meily, Caroline M. van Heugten, Tanja C. W. Nijboer. Acquisition of data: Lauriane A. Spreij. Analysis of data: Lauriane A. Spreij, Tanja C. W. Nijboer. Interpretation of data: Lauriane A. Spreij, Tanja C. W. Nijboer. Drafting and revising the work: Lauriane A. Spreij, Johanna M. A. Visser-Meily, Caroline M. van Heugten, Tanja C. W. Nijboer. Final approval of the version to be published: Lauriane A. Spreij, Johanna M. A. Visser-Meily, Caroline M. van Heugten, Tanja C. W. Nijboer. Agreement to be accountable for all aspects of the work: Lauriane A. Spreij, Johanna M. A. Visser-Meily, Caroline M. van Heugten, Tanja C. W. Nijboer.

\section{ACKNOWLEDGMENTS}

Tanja C. W. Nijboer is supported by the NWO (Netherlands Organization for Scientific Research; grant 451-10-013).

\section{REFERENCES}

Aben, L., Heijenbrok-Kal, M. H., van Loon, E. M. P., Groet, E., Ponds, R. W. H. M., Busschbach, J. J. V., et al. (2013). Training memory self-efficacy in the chronic stage after stroke: a randomized controlled trial. Neurorehabil. Neural Repair 27, 110-117. doi: 10.1177/1545968312455222

Åkerlund, E., Esbjörnsson, E., Sunnerhagen, K. S., and Björkdahl, A. (2013). Can computerized working memory training improve impaired working memory, cognition, and psychological health? Brain Inj. 27, 13-14. doi: 10.3109/02699052.2013.830195

Björkdahl, A., Åkerlund, E., Svensson, S., and Esbjörnsson, E. (2013). A randomized study computerized working memory training and effects on functioning in everyday life for patients with brain injury. Brain Inj. 27, 1658-1665. doi: 10.3109/02699052.2013.830196

Brooks, B. M., and Rose, F. D. (2003). The use of virtual reality in memory rehabilitation: current findings and future directions. Neurorehabilitation 18, 147-157.

Brooks, B. M., Rose, F. D., Potter, J., Jayawardena, S., and Morling, A. (2004). Assessing stroke patients'prospective memory using virtual reality. Brain Inj. 18, 391-401. doi: 10.1080/02699050310001619855

Brunoni, A. R., and Vanderhasselt, M.-A. (2014). Working memory improvement with non-invasive brain stimulation of the dorsolateral prefrontal cortex: a systematic review and meta-analysis. Brain Cogn. 86, 1-9. doi: 10.1016/j.bandc.2014.01.008

Caglio, M., Latini-Corazzini, L., D’Agata, F., Cauda, F., Sacco, K., Monteverdi, S., et al. (2012). Virtual navigation for memory rehabilitation in a traumatic brain injured patient. Neurocase 18, 123-131. doi: 10.1080/13554794.2011.568499 
Cappa, S. F., Benke, T., Clarke, S., Rossi, B., Stemmer, B., and van Heugten, C. M. (2011). Cognitive rehabilitation. Euro. Handb. Neurol. Manag. 1, 545-567. doi: 10.1002/9781444328394.ch40

Cicerone, K. D., Langenbahn, D. M., Braden, C., Malec, J. F., Kalmar, K., Fraas, M., et al. (2011). Evidence-based cognitive rehabilitation: updated review of the literature from 2003 through 2008. Arch. Phys. Med. Rehab. 92, 519-530. doi: 10.1016/j.apmr.2010.11.015

Cramer, S. C. (2008). Repairing the human brain after stroke: 1. Mechanisms of spontaneous recovery. Ann. Neurol. 63, 272-287. doi: 10.1002/ana.21393

Das Nair, R., and Lincoln, N. (2007). Cognitive rehabilitation for memory deficits following stroke. Cochrane Database of Syst. Rev. 3, 1-15. doi: 10.1002/14651858. CD002293.pub2

De Luca, R., Calabrò, R. S., Gervasi, G., De Salvo, S., Bonanno, L., Corallo, F., et al. (2014). Is computer-assisted training effective in improving rehabilitative outcomes after brain injury? A case-control hospital-based study. Disabil. Health J. 7, 356-360. doi: 10.1016/j.dhjo.2014.04.003

Fasotti, L., and van Kessel, M. (2013). Novel insights in the rehabilitation of neglect. Front. Hum. Neurosci. 7:780. doi: 10.3389/fnhum.2013.00780

Fish, J., Manly, T., Emslie, H., Evans, J. J., and Wilson, B. A. (2008). Compensatory strategies for acquired disorders of memory and planning: differential effects of a paging system for patients with brain injury of traumatic versus cerebrovascular aetiology. J. Neurol. Neurosurg. Psychiatry 79, 930-935. doi: 10.1136/jnnp.2007.125203

Hall, R. C. W., Hall, R. C. W., and Capman, M. J. (2005). Definition, diagnosis, and forensic implications of postconcussional syndrome. Psychosomatics 43, 195-202. doi: 10.1176/appi.psy.46.3.195

Holmqvist, K., Kamwendo, K., and Ivarsson, A. B. (2009). Occupational therapists' descriptions of their work with persons suffering from cognitive impairment following acquired brain injury. Scand. J. Occup. Ther. 16, 13-24. doi: 10.1080/11038120802123520

Jo, J. M., Kim, M. H., Ohn, S. H., Joen, B., and Lee, K. H. (2009). Enhancing the working memory of stroke patients using tDCS. Am. J. Psys. Med. Rehabil. 88, 404-409. doi: 10.1097/PHM.0b013e3181a0e4cb

Johansson, B. B. (2011). Current trends in stroke rehabilitation: a review with focus on Brain Plasticity. Acta Neurol. Scand. 123, 147-159. doi: 10.1111/j.16000404.2010.01417.x

Johansson, B. B., and Tornmalm, M. (2012). Working memory training for patients with acquired brain injury: effects in daily life. Scand. J. Occup. Ther. 19, 176-183. doi: 10.3109/11038128.2011.603352

Kessels, R. P. C., d'Alfonso, A. A. L., Postma, A., and de Haan, E. H. F. (2000). Spatial working memory performance after high-frequency repetitive transcranial magnetic stimulation of the left and right posterior parietal cortex in humans. Neurosci. Lett. 287, 68-70. doi: 10.1016/S0304-3940(00) 01146-0

Kim, B. R., Kim, D. Y., Chun, M. H., Yi, J. H., and Kwon, J. S. (2010). Effect of repetitive transcranial magnetic stimulation on cognition and mood in stroke patients: a double-blind, sham-controlled trial. Am. J. Phys. Med. Rehabil. 89, 362-368. doi: 10.1097/PHM.0b013e3181d8a5b1

Kimberley, T., Samargia, S., Moore, L., Shakya, J. K., and Lang, C. E. (2010). Comparison of amounts and types of practice during rehabilitation for traumatic brain injury and stroke. J. Rehabil. Res. Dev. 47, 851-861. doi: 10.1682/JRRD.2010.02.0019

Klingberg, T. (2010). Training and plasticity of working memory. Trends Cogn. Sci. 14, 317-324. doi: 10.1016/j.tics.2010.05.002

Leśniak, M., Polanowska, K., Seniów, J., and Członkowska, A. (2014). Effects of repeated anodal tDCS coupled with cognitive training for patients with severe traumatic brain injury: a pilot randomized controlled trial. J. Head Trauma Rehabil. 29, 20-29. doi: 10.1097/HTR.0b013e318292a4c2

Levine, B., Fugiwara, E., O’Connor, C., Richard, N., Kovacevic, N., Mandic, M., et al. (2006). In vivo characterization of traumatic brain injury neuropathology with structural and functional neuroimaging. J. Neurotrauma 23, 1396-1441. doi: 10.1089/neu.2006.23.1396

Li, K., Robertson, J., Ramos, J., and Gella, S. (2013). Computer-based cognitive retraining for adults with chronic acquired brain injury: a pilot study. Occup. Ther. Health Care 27, 333-344. doi: 10.3109/07380577.2013. 844877

Lim, J. Y., Kang, E. K., and Paik, N. J. (2010). Repetitive transcranial magnetic stimulation to hemispatial neglect in patients after stroke: an open-label pilot study. J. Rehabil. Med. 42, 447-452. doi: 10.2340/16501977-0553
Luber, B., Kinnunen, L. H., Rakitin, B. C., Ellsasser, R., Stern, Y., and Lisanby, S. H. (2007). Facilitation of performance in a working memory task with rTMS stimulation of the precuneus: frequency- and time-dependent effects. Brain Res. 1128, 120-129. doi: 10.1016/j.brainres.2006.10.011

Lundqvist, A., Grundström, K., Samuelsson, K., and Rönnberg, J. (2010). Computerized training of working memory in a group of patients suffering from acquired brain injury. Brain Inj. 24, 1173-1183. doi: 10.3109/02699052.2010.498007

Miniussi, C., Cappa, S. F., Coohen, L., Floel, A., Fregni, F., Nitsch, M. A., et al. (2008). Efficacy of repetitive transcranial magnetic stimulation/transcrianial direct current stimulation in cognitive neurorehabilitation. Brain Stimul. 1, 326-336. doi: 10.1016/j.brs.2008.07.002

Naeser, M. A., Martin, P. I., Nicholas, M., Baker, E. H., Seekins, H., Kobayashi, M., et al. (2005). Improved picture naming in chronic aphasia after TMS to part of right Broca's area: an open protocol study. Brain Lang. 93, 95-105. doi: 10.1016/ j.bandl.2004.08.004

Nyffeler, T., Cazzoli, D., Hess, C. W., and Muri, R. M. (2009). One session of repeated parietal theta burst stimulation trains induces long-lasting improvement of visual neglect. Stroke 40, 2791-2796. doi: 10.1161/STROKEAHA.109.552323

Olesen, P. J., Westerberg, H., and Klingberg, T. (2004). Increased prefrontal and parietal activity after training of working memory. Nat. Neurosci. 7, 75-79. doi: 10.1038/nn1165

Oliveri, M., Turriziani, P., Carlesimo, G. A., Koch, G., Tomaiuolo, F., Panella, M., et al. (2001). Parieto-frontal interactions in visual-object and visual-spatial working memory: evidence from transcranial magnetic stimulation. Cereb. Cortex 11, 606-618. doi: 10.1093/cercor/11.7.606

Optale, G., Urgesi, C., Busato, V., Piron, L., Priftis, K., Gamberini, L., et al. (2009). Controlling memory impairment in elderly adults using virtual reality memory training; a randomized controlled pilot study. Neurorehabil. Neural Repair 24, 348-357. doi: 10.1177/1545968309353328

Park, S.-H., Koh, E.-J., Choi, H.-Y., and Ko, M.-H. (2013). A double-blind, shamcontrolled, pilot study to assess the effects of the concomitant use of transcranial direct current stimulation with the computer assisted cognitive rehabilitation to the prefrontal cortex on cognitive functions in patients with stroke. J. Korean Neurosurg. Soc. 54, 484-488. doi: 10.3340/jkns.2013.54.6.484

Park, S. H., Seo, J. H., Kim, Y., and Ko, M. H. (2014). Long-term effects of transcranial direct current stimulation combined with computer-assisted cognitive training in healthy older adults. Neuroreport 25, 122-126. doi: 10.1097/WNR.0000000000000080

Preston, G., Anderson, E., Silva, C., Goldberg, T., and Wassermann, E. M. (2010). Effects of $10 \mathrm{~Hz}$ rTMS on the neural efficiency of working memory. J. Cogn. Neurosci. 22, 447-456. doi: 10.1162/jocn.2009.21209

Rees, L., Marshall, S., Hartridge, C., Mackie, D., and Weiser, M. (2007). Cognitive interventions post acquired brain injury. Brain Inj. 21, 161-200. doi: $10.1080 / 02699050701201813$

Schrijnemaekers, A.-C., Smeets, S. M. J., Ponds, R. W. H., van Heugten, C., and Rasquin, S. (2013). Treatment of unawareness of dificits in patients with acquired brain injury: a systematic review. J. Head Trauma Rehabil. 29, 1-21. doi: 10.1097/01.HTR.0000438117.63852.b4

Snaphaan, L., and de Leeuw, F.-E. (2007). Poststroke memory function in non-demented patients: a systematic review on frequency and neuroimaging correlates. Stroke 38, 198-201. doi: 10.1161/01.STR.0000251842. 34322.8f

Szaflarski, J. P., Vannest, J., Wu, S. W., DiFrancesco, M. W., Banks, C., and Gilbert, D. L. (2011). Excitatory repetitive transcranial magnetic stimulation induces improvements in chronic post-stroke aphasia. Med. Sci. Monit. 17, 132-139. doi: 10.12659/MSM.881446

Tijssen, J. G. P., and Assendelft, W. J. J. (2003). "Beoordeling van een gerandomiseerd effectonderzoek," in Inleiding in Evidence-Based Medicine, eds M. Offringa, W. J. J. Assendelft, and R. J. P. M. Scholten (Houten: Bohn Stafleu van Loghum), 56-70.

Ulam, F., Shelton, C., Richards, L., Davis, L., Hunter, B., Fregni, F., et al. (2014). Cumulative effects of transcranial direct current stimulation on EEG oscillations and attention/working memory during subacute neurorehabilitation of traumatic brain injury. Clin. Neurophysiol. doi: 10.1016/j.clinph.2014.05.015. [Epub ahead of print].

Villamar, M. F., Portilla, A. S. P., Fregni, F., and Zafonte, R. (2012). Nonivasive Brain stimulation to modulate neuroplasticity in traumatic 
brain injury. Neuromodulation 15, 326-338. doi: 10.1111/j.1525-1403.2012. 00474.x

Westerberg, H., Jacobaeus, H., Hirvikoski, T., Clevberger, P., Ostensson, M.-L., Bartfai, A., et al. (2007). Computerized working memory training after stroke a pilot study. Brain Inj. 21, 21-29. doi: 10.1080/02699050601148726

Yip, B. C. B., and Man, D. W. K. (2009). Virtual reality-based prospective memory training program for people with acquired brain injury. Neurorehabilitation 32, 103-115. doi: 10.3233/NRE-130827

Yip, B. C. B., and Man, D. W. K. (2013). Virtual reality (VR)-based community living skills training for people with acquired brain injury: a pilot study, Brain Inj. 23, 1017-1026. doi: 10.3109/02699050903 379412

You, S. H., Jang, S. H., Kim, Y. H., Hallett, M., Ahn, S. H., Kwon, Y. H., et al. (2005). Virtual Reality-induced cortical reorganization and associated locomotor recovery in chronic stroke: an experimenter-blind randomized study. Stroke 36, 1166-1171. doi: 10.1161/01.STR.0000162715. 43417.91
Conflict of Interest Statement: The authors alone are responsible for the content and writing of this review. The authors declare that the research was conducted in the absence of any commercial or financial relationships that could be construed as a potential conflict of interest.

Received: 08 October 2014; paper pending published: 06 November 2014; accepted: 22 November 2014; published online: 16 December 2014.

Citation: Spreij LA, Visser-Meily JMA, van Heugten CM and Nijboer TCW (2014) Novel insights into the rehabilitation of memory post acquired brain injury: a systematic review. Front. Hum. Neurosci. 8:993. doi: 10.3389/fnhum.2014.00993 This article was submitted to the journal Frontiers in Human Neuroscience.

Copyright () 2014 Spreij, Visser-Meily, van Heugten and Nijboer. This is an openaccess article distributed under the terms of the Creative Commons Attribution License (CC BY). The use, distribution or reproduction in other forums is permitted, provided the original author(s) or licensor are credited and that the original publication in this journal is cited, in accordance with accepted academic practice. No use, distribution or reproduction is permitted which does not comply with these terms. 